\title{
Políticas educativas del Consorcio de Educación de Barcelona en relación al aprendizaje-servicio como innovación social
}

\section{Francesc Vila Batallé}

Área de Innovación, Programas y Formación del profesorado, Consorcio de Educación de Barcelona

\section{Resumen}

A lo largo de la breve historia del Consorcio de Educación de Barcelona, el aprendizaje-servicio siempre ha estado presente entre los programas de innovación que se imparten en los centros educativos de la ciudad. Durante estos dos últimos años, y con la voluntad de impulsar la transformación educativa, se ha repensado el perfil de los profesionales de los centros de recursos pedagógicos, se han reforzado los vínculos de colaboración con los diferentes organismos municipales y entidades interesadas, así como se ha creado la convocatoria unificada de programas.

\section{Palabras clave}

Aprendizaje-servicio, innovación, transformación educativa, centros de recursos pedagógicos. 


\title{
Educational policies of the Barcelona Education Consortium in relation to service-learning as a social innovation
}

\author{
Abstract \\ Throughout the brief history of the Barcelona Education Consortium, service-learning \\ has always been present among the innovative programmes taught in the city's \\ educational centres. Over the past two years, and with the aim to promote \\ educational transformation, the profile of the professionals at the Pedagogical \\ Resource Centres has been rethought; collaboration links with different municipal \\ organisms and interested parties have been strengthened, and a unified call for \\ programmes has been made
}

\section{Keywords}

Service-learning, innovation, educative transformation, pedagogical resource centres. 


\section{1. ¿Quiénes somos?}

El Consorcio de Educación de Barcelona se constituye en el año 2002 con la voluntad de crear un marco de cooperación entre estructuras públicas y con el objetivo de mejorar la gestión educativa de la ciudad y hacerla más próxima al ciudadano. Se trata pues, de un instrumento de cogestión y descentralización, en un marco de colaboración institucional, que expresa la voluntad del gobierno de la Generalitat de Catalunya y del Ayuntamiento de Barcelona de trabajar conjuntamente para la mejora de los servicios en los centros educativos a través de una única estructura. Es decir, se crea un organismo a través del cual la Generalitat de Catalunya y el Ayuntamiento de Barcelona comparten esfuerzos para gestionar de manera unificada las funciones, actividades y servicios de la ciudad en materia educativa, relacionada con todos los niveles de enseñanza no universitaria. De hecho, la creación del Consorcio de Educación es una respuesta actual que conecta con la tradición innovadora de la ciudad en el terreno de la educación $y$, al mismo tiempo, en coherencia con las nuevas formas de cooperación y gestión pública, las cuales buscan la suma de esfuerzos, la creación de un marco de gestión compartida y la coordinación efectiva evitando la duplicidad de servicios.

Entre sus muchas competencias y funciones en el marco de la planificación general está la creación, la gestión y la organización de los centros pedagógicos y de recursos de servicios de soporte, asesoramiento e investigación educativa, así como la formación permanente del personal docente.

En el organigrama del Consorcio figura la Dirección de Acción Educativa que consta de cuatro áreas, una de las cuales es la de Innovación, Programas y Formación del profesorado. Ésta, a su vez, engloba a los diez Centros de Recursos Pedagógicos de la ciudad y al Equipo de Lengua y Cohesión Social, recursos y servicios educativos repartidos por la ciudad que descentralizan las acciones educativas del Consorcio favoreciendo un trabajo de proximidad en cada uno de los diez distritos de la ciudad.

\section{2. ¿Qué estamos haciendo?}

El Consorcio de Educación de Barcelona ha apostado por replantear los nuevos perfiles de los profesionales de los centros de recursos pedagógicos como asesores de innovación y como dinamizadores de redes de transformación educativa.

Desde el Área de Innovación se quiere avanzar, a través de los profesionales de los centros de recursos pedagógicos, hacia un servicio educativo de asesores, redefiniendo los servicios de soporte que ya desarrollan en los centros educativos. Por lo tanto, al margen del asesoramiento y soporte de contenido o especialización técnica, el perfil de asesores en innovación educativa contempla la dinamización y acompañamiento en los procesos de transformación y mejora educativa de los centros. Este perfil profesional también contempla la dinamización de redes de profesorado, equipos

Vila, F. (2018). Políticas educativas del Consorcio de Educación de Barcelona en relación al aprendizajeservicio como innovación social. RIDAS, Revista Iberoamericana de Aprendizaje Servicio, 6, 33-41. DOI10.1344/RIDAS2018.6.5 
directivos y especialistas docentes así como el perfil de mentor, un consejero o guía que apadrina a los centros que lo soliciten en su proceso de transformación educativa. Es importante la capacidad de trabajo en red con otros centros para contrastar las experiencias pedagógicas que se realizan compartiendo procesos de reflexión y formación o bien actuar de referentes para otros.

El rol de este mentor se lleva a cabo a través de un programa propio del Consorcio de Educación Ilamado Herramientas para el Cambio, basado en el acompañamiento personalizado al centro por parte del mentor con el fin de reforzar el proceso de transformación educativa por el cual se opta y con el objetivo que en el centro se promocione una comunidad de práctica reflexiva, donde la mejora educativa se centre en el proceso de aprendizaje. También el Consorcio dinamiza el programa Redes para el Cambio en convenio con Escola Nova 21, el Instituto de Ciencias de la Educación de la Universidad Autónoma de Barcelona y la Asociación de Maestros Rosa Sensat, con el reto que en tres años todos los centros educativos de la ciudad puedan estar inmersos en algún proyecto de mejora sostenible desde el trabajo en red.

Desde los centros de recursos pedagógicos y el Área de Innovación, Programas y Formación se gestionan los diferentes programas de innovación educativa y la formación del profesorado de la ciudad que, generalmente, acostumbra a ir asociada a uno de estos programas. Algunos de los que se están dinamizando actualmente son Herramientas para el cambio, Redes para el cambio, Exit, Enginy, Impulso a la lectura, LiterapolisBCN, Clubs de lectura aumentada, Generación plurilingüe, Talk to me, Avancemos hacia el tratamiento integrado de lenguas (plurilingüismo), Erasmus+, mSchools Scratch, STEAM en las aulas, Congreso de ciencia, Ciencia ciudadana, Escuelas más sostenibles, Patrimoníame, Magnet, Tándem, Explícanos el barrio, Convivencia en los centros escolares, Why violence?, Escuelas para la igualdad i la diversidad, Mediación entre iguales, yAprendizaje-servicio entre muchos otros.

A finales del curso escolar 2017-2018 se ha creado una convocatoria unificada de programas con la voluntad de armonizar toda la oferta de programas educativos que imparten los centros, unificar los calendarios y garantizar la calidad de la propuesta metodológica, la transparencia en los criterios de asignación y la coherencia con el proyecto educativo de centro, poniendo al alcance de las escuelas y institutos un mapa de alianzas que ayude a avanzar hacia una oferta coherente de programas educativos.

Esta convocatoria unificada pretende facilitar la programación general anual de cada centro y evitar el goteo continuado de propuestas de programas educativos a lo largo del curso, de manera que éstos se inserten en una secuencia lógica de planificación y de trabajo para la transformación educativa del mismo.

Los centros de recursos pedagógicos, 
después de trabajar con ellos el proceso de apropiación, se están atreviendo a pilotar y estimular el cambio pedagógico, actuando y trabajando en redes colaborativas entre centros de una misma zona y ejerciendo de mentores, tomando decisiones a partir de evidencias e investigaciones. Si partimos de la base que el modelo educativo tradicional está agotado, tenemos que asumir las nuevas funciones sociales de los centros educativos, es decir, innovación y cambio hacia un nuevo paradigma.

Este ejercicio de repensar y redefinir las funciones y perfiles profesionales de los centros de recursos pedagógicos hacia un nuevo modelo de trabajo se ha efectuado a través del debate con los diferentes profesionales, una formación interna y acompañamiento de profesionales externos. Además, se ha llevado a término la especialización temática de tres de ellos muy ligados a la innovación educativa. Son las tecnologías para el aprendizaje y el conocimiento en Ciutat Vella, las enseñanzas artísticas (música, danza, teatro y artes plásticas) en Sant Andreu y ciudadanía y valores en Les Corts. El objetivo es crear unos espacios de referencia en la ciudad donde la concentración de especialistas pueda facilitar la producción colaborativa de contenidos, la visibilidad del servicio y la modelización de una tipología de espacios y recursos determinados.

Esta especialización no significa en ningún momento tener que renunciar al trabajo de proximidad en el territorio, ya que los referentes de cada uno de estos temas en cada centro de recursos pedagógicos continúan existiendo. Es decir, el papel de liderazgo del centro de recursos pedagógicos Les Corts en ciudadanía y valores hace que los referentes de cada centro de recursos pedagógicos en aprendizaje-servicio se reúnan periódicamente para crear un discurso común de ciudad y contemplar a la vez la singularidad del distrito y de cada uno de los barrios. La gran ventaja es que se trabaja con un equipo de profesionales más amplio por lo que se genera mucho más conocimiento experto.

En relación a esto, cabe destacar tres ejes de actuación del centro de recursos pedagógicos Les Corts: documentación e investigación, divulgación y formación. Éste debe convertirse en un buen centro de documentación recogiendo toda la información posible para abordar el trabajo de ciudadanía y valores, por consiguiente también de aprendizajeservicio, en los centros educativos de la ciudad.

El centro de recursos pedagógicos Les Corts deberá contribuir a divulgar las actividades y recursos de la ciudad, tanto las puntuales como las de larga duración. También tendrá que hacer difusión de los trabajos del alumnado y proyectos e investigaciones del profesorado buscando ampliar y crear nuevos canales de publicación acercando los diferentes medios y canales de comunicación a los productos.

En cuanto a la formación del profesorado, una de las líneas de acción prioritarias de los centros de recursos pedagógicos se ha de vincular al modelo educativo del siglo XXI, por

Vila, F. (2018). Políticas educativas del Consorcio de Educación de Barcelona en relación al aprendizajeservicio como innovación social. RIDAS, Revista Iberoamericana de Aprendizaje Servicio, 6, 33-41. 
tanto los contenidos de ciudadanía y valores han de ser transversales en las diferentes áreas del currículo a través del trabajo por proyectos, aunque es sabido que el currículo puede ser el enemigo de la innovación. También ha de servir para establecer modelos didácticos a la vez que plantea nuevas perspectivas y formas de trabajar ayudando a descubrir los recursos del barrio y la ciudad.

La educación ha de desarrollar competencias para la vida, por lo que nos obliga a repensar el propósito de la educación en base a las necesidades sociales y la organización del aprendizaje en base al conocimiento que tenemos de cómo aprendemos las personas, cosa que nos permite ser creativos y transformar para generar cambio. $Y$ es aquí donde el aprendizaje-servicio tiene un papel fundamental y se convierte en un gran aliado para avanzar en la transformación educativa.

Pero nunca tenemos que perder de vista que el alumnado es el verdadero protagonista del cambio, pues adopta un papel dentro de la sociedad que antes no tenía siendo muchas veces un ejemplo para los otros a través del efecto contagio a pequeños y mayores. Es muy importante poner la acción tutorial del alumnado en este acompañamiento como partícipe en actividades de educación no formal, haciendo que se sienta muy bien orientado desde la formación hasta la valoración de la propia práctica; conseguir que se sienta parte de lo público, preparado para decirnos cosas y también transformarnos. Así mismo, es necesario dar protagonismo al alumnado en la detección de las necesidades del entorno para llevar a cabo un servicio y para que sea autónomo y pueda elegir.

Es cierto que se nos plantea una dificultad con la exigencia de convertir el aprendizaje-servicio en obligatorio a partir del curso 2019-2020 para el alumnado de segundo ciclo de la Educación Secundaria Obligatoria $\left(3^{\circ}\right.$ y $4^{\circ}$ curso), porque exigirá más oferta cualitativa y cuantitativa de proyectos. En cuanto a la calidad de proyectos, podemos replantear la organización de los centros de secundaria sobre todo en relación a la agrupación de alumnos, la manera de reforzar los aprendizajes y las competencias a trabajar, más allá de los valores y la implicación de más materias.

Para el Consorcio de Educación, el aprendizaje-servicio siempre ha estado presente entre los programas de innovación educativa que se llevan a cabo en los centros de la ciudad, por lo que no le resulta tan novedoso. Desde los inicios de su creación, el Consorcio ha colaborado con diferentes entidades para aproximar el aprendizaje, en los centros educativos de todas las etapas, haciendo un servicio a la comunidad.

Cabe resaltar que será obligatorio para los centros educativos, no así para las entidades, hecho que provoca un desajuste por lo que éstas necesitan un reto estimulante más allá de la convocatoria de ayudas y la formación de sus miembros. Se trata de proponer proyectos fáciles para ampliar la base y más adelante ya se construirá la complejidad de éstos.

Vila, F. (2018). Políticas educativas del Consorcio de Educación de Barcelona en relación al aprendizajeservicio como innovación social. RIDAS, Revista Iberoamericana de Aprendizaje Servicio, 6, 33-41. 


\section{3. ¿Con quién colaboramos?}

Un reto con el que se está trabajando es el de encontrar el encaje a nivel político con la propuesta de ciudad educadora, una gran oportunidad de actualizar un discurso común construido conjuntamente. Para ello contamos con el soporte de diferentes entidades y organismos municipales con los que estamos trabajando: la Dirección de Justicia Global y Cooperación Internacional, el Consejo de Innovación Pedagógica -impulsado por el Instituto Municipal de Educación de Barcelona-, y la red de Escuelas sostenibles. Éstas últimas tienen como objetivo contribuir a la sostenibilidad desde la educación y con el reconocimiento de las escuelas e institutos de la ciudad como agentes de cambio.

La Dirección de Justicia Global y Cooperación Internacional, entre otras muchas actuaciones, se encarga de la convocatoria de concurso público para la concesión de subvenciones en el marco del Programa de Cooperación para la Justicia Global, el Programa de Cooperación para la Justicia Global en Ciudades Específicas y el Programa de Educación para la Justicia Global. La finalidad de estas subvenciones es la de aportar los recursos necesarios para el cumplimiento de los objetivos estratégicos fijados en el Plan Director de Cooperación al Desarrollo, Solidaridad y Paz, vigente en el momento de aprobación de la convocatoria por la Comisión de Gobierno. $Y$ estas subvenciones han dado un gran impulso a las entidades en relación al aprendizaje-servicio.
Estas entidades de justicia global y cooperación internacional son agentes educativos con capacidad de transformación por lo que se trata de un valor añadido como se puede leer en las bases de la convocatoria.

Además del soporte económico, están colaborando mucho con el asesoramiento a las entidades creando una construcción conjunta del discurso entre las organizaciones no gubernamentales y la escuela, más allá de que los centros adquieran un proyecto. Esta idea se refuerza con la participación en un seminario de intercambio de proyectos, dirigido a las entidades, con un componente formativo muy importante.

También cabe mencionar a Lafede.cat organizaciones para la justicia globalcomo red que promueve la acción colectiva de las 116 organizaciones federadas y que trabaja activamente para conseguir la justicia social y la erradicación de las desigualdades mediante la cooperación al desarrollo, la defensa y la promoción de los derechos humanaos y el fomento de la paz.

Se ha trabajado mucho a nivel de ciudad con la Dirección de Justicia Global y Cooperación Internacional y ahora toca hacerlo a nivel de territorio por lo que, con el objetivo de recoger las prácticas de Educación para la Justicia Global existentes en la ciudad de Barcelona, se ha diseñado un mapa interactivo en el que se puede consultar la información básica de estos proyectos. Un mapa que complementa la información relacionada con los 
proyectos de aprendizaje servicio recogidos en la web del Consorcio. Además, se ha creado a nivel municipal la figura del referente de justicia global en cada uno de los distritos, un gran aliado con el referente de aprendizajeservicio en cada uno de los centros de recursos pedagógicos.

Por otro lado, las entidades no sociales también toman un compromiso con la ciudadanía, de ahí la colaboración entre el Consorcio y el Consejo de Innovación Pedagógica. El objetivo del Consejo de Innovación Pedagógica del Ayuntamiento de Barcelona es garantizar la mejora de las diferentes propuestas educativas, promoviendo la innovación a través de metodologías pedagógicas, el compromiso social y la creación de nuevos espacios de intercambio y conocimiento. La red del Consejo de Innovación Pedagógica la forman en este momento 159 instituciones y entidades ciudadanas que ofrecen más de 5.100 actividades educativas, por medio del Programa de Actividades Escolares a centros educativos de la ciudad de Barcelona y de toda Cataluña.

El Consejo de Innovación Pedagógica parte de la visión de las entidades ofreciendo una red de instituciones, la prioridad de muchas de las cuales no es educativa, pero que a la vez enriquecen las dimensiones educativas de las escuelas e institutos.

Al disponer de tiempo, se pueden ir incorporando nuevas tipología de entidades más allá de las que ya hacen aprendizaje-servicio, para que la dimensión comunitaria tenga un sentido más amplio. Además, lo fundamental es que el servicio sea significativo y es muy importante focalizarlo para no correr el riesgo que el aprendizaje, del que se ha hecho mucha investigación, sea mucho más visible que el servicio. En este sentido, tenemos la suerte que la dimensión de la ciudad ofrece la posibilidad de colaborar con muchas entidades cosa que nos ha ayudado a significar las actividades más allá de las campañas.

Como novedad, este año, y con la esperanza de repetir sucesivamente, se ha realizado por primera vez un seminario para el análisis y mejora de proyectos con entidades del Consejo de innovación y centros de secundaria del Consorcio dinamizado por el Centro Promotor del ApS con la finalidad de abrir un espacio de encuentro, reflexión y construcción conjunta entre entidades y centros educativos que ya tienen una experiencia previa en aprendizajeservicio y que comparten la voluntad de mejorar sus propuestas y proyectos.

Esta metodología pedagógica es la intervención local en materia educativa, el medio para que pasen cosas por su propuesta educativa. Así pues, remarcar el poder de la parte municipal en el aprendizaje-servicio nos hace repensar el papel de la ciudadanía en el ideal de ciudad educadora. Nos permite ser creativos y transformar para generar cambio buscando aliados fuera del centro y estableciendo redes de cooperación, a parte que aprendemos más y mejor cuando trabajamos juntos ¿Quién está bien posicionado para hacer transformación? Los centros educativos, las entidades y los agentes

Vila, F. (2018). Políticas educativas del Consorcio de Educación de Barcelona en relación al aprendizajeservicio como innovación social. RIDAS, Revista Iberoamericana de Aprendizaje Servicio, 6, 33-41. 


\section{comunitarios}

El hecho que el Consorcio de Educación tenga la suma del poder local y del gobierno de la Generalitat, facilita el encaje ya que su naturaleza mixta nos permite trabajar en la misma dirección, acompañando el proceso pasando de lo aislado a lo estratégico.

Y en esta línea estratégica de arraigar en el territorio, se ha materializado la colaboración, para el curso 2018-2019, con el Centro Promotor de AprendizajeServicio y la Dirección de Justicia Global y Cooperación Internacional con la creación de un plan de implementación del aprendizajeservicio en los diez distritos de la ciudad de Barcelona. Un plan con un doble objetivo: consolidar las experiencias y proyectos de aprendizaje-servicio que se están llevando a cabo, incentivar de nuevas y promover algunas de alta calidad pedagógica así como crear en cada distrito las condiciones necesarias para conseguir el arraigo, la sostenibilidad y la multiplicación del aprendizajeservicio. Para conseguirlo se ha diseñado conjuntamente un seminario de reflexión y planificación dirigido a los referentes de aprendizaje servicio que hay en cada uno de los centros de recursos pedagógicos como dinamizadores de los diferentes proyectos de los centros educativos de sus territorios, los técnicos referentes de justicia global de cada uno de los distritos y algunos miembros referentes del aprendizaje servicio del Consejo de Innovación Pedagógica y de las Escuelas sostenibles.
Esta colaboración contempla también, en la línea del trabajo de proximidad en el territorio, una serie de acciones puntuales en cada uno de los distritos, a demanda de los agentes que intervienen, para facilitar la implementación del aprendizajeservicio, promover jornadas de intercambio entre centros, potenciar los actos de reconocimiento del alumnado o incluso ayudar en la dinamización y formación (inicial o de actualización) del profesorado. Unas acciones que se diseñaran previamente en el seminario con la implicación de todos los participantes.

Es importante destacar que, en relación al aprendizaje-servicio, tenemos un horizonte compartido para toda la ciudad. Desde el Consorcio de Educación de Barcelona se continuará trabajando para arraigar y consolidar, a través de los centros de recursos pedagógicos y con los diferentes organismos municipales colaboradores, todo el trabajo que esta propuesta educativa nos ofrece para hacer posible que la innovación se produzca en el centro educativo. 